\title{
Nota
}

\section{REMEDIAÇÃO DE UM SOLO CONTAMINADO COM PETRÓLEO POR OXIDAÇÃO QUÍMICA ${ }^{(1)}$}

\author{
Vivian Andrade Graciano ${ }^{(2)}$, Carolina de Souza Pires ${ }^{(2)}$, Silvio César Godinho Teixeira ${ }^{(3)}$, \\ Roberta Lourenço Ziolli ${ }^{(4)}$ \& Daniel Vidal Pérez ${ }^{(5)}$
}

\begin{abstract}
RESUMO
A remediação de uma amostra de Latossolo Vermelho-Amarelo contaminado artificialmente com petróleo $\left(5.000 \mathrm{mg} \mathrm{kg}^{-1}\right)$ foi estudada comparando diferentes oxidantes químicos: $\mathrm{KMnO}_{4} 0,10 \mathrm{~mol} \mathrm{~L}^{-1} ; \mathrm{K}_{2} \mathrm{~S}_{2} \mathrm{O}_{8} 0,10 \mathrm{~mol} \mathrm{~L}^{-1} ; \mathrm{H}_{2} \mathrm{O}_{2} 0,10 \mathrm{~mol} \mathrm{~L}^{-1}$;

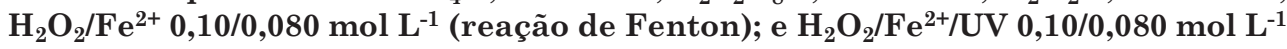
(reação de foto-Fenton). A remediação foi praticamente completa aos $30 \mathrm{~min}$ para o processo de foto-Fenton (99\%). Todos os outros oxidantes não atingiram essa taxa de dissipação até $180 \mathrm{~min}$. $O$ processo em que se usou $\mathrm{H}_{2} \mathrm{O}_{2} 0,10 \mathrm{~mol} \mathrm{~L}^{-1}$ $\mathbf{( 7 0 , 5 1 \% )}$ foi o de menor eficiência. A adição de íons Fe aumentou a taxa de dissipação para $86,98 \%\left(\mathrm{H}_{2} \mathrm{O}_{2} / \mathrm{Fe}^{2+} 0,10 / 0,080 \mathrm{~mol} \mathrm{~L}^{-1}\right)$. Os métodos convencionais apresentaram taxas de dissipação de 76,58 \% $\left(\mathrm{KMnO}_{4} \mathbf{0 , 1 0} \mathrm{mol} \mathrm{L}^{-1}\right)$ e $93,85 \%$ $\left(\mathrm{K}_{2} \mathrm{~S}_{2} \mathrm{O}_{8} 0,10 \mathrm{~mol} \mathrm{~L}^{-1}\right)$.
\end{abstract}

Termos de indexação: reação de Fenton, peróxido, permanganato, persulfato.

\section{SUMMARY: REMEDIATION OF OIL-CONTAMINATED SOIL BY CHEMICAL OXIDATION}

The possibilities of remediation of a Latossolo Vermelho Amarelo artificially contaminated with oil (5,000 $\left.\mathrm{mg} \mathrm{kg}^{-1}\right)$ were investigated. Different remediation methods with chemical oxidants were compared: $\mathrm{KMnO}_{4} 0.10 \mathrm{~mol} \mathrm{~L}^{-1} ; \mathrm{K}_{2} \mathrm{~S}_{2} \mathrm{O}_{8} 0.10 \mathrm{~mol} \mathrm{~L}^{-1} ; \mathrm{H}_{2} \mathrm{O}_{2} 0.10 \mathrm{~mol} \mathrm{~L}^{-1} ; \mathrm{H}_{2} \mathrm{O}_{2} / \mathrm{Fe}^{2+} 0.10$ / $0.08 \mathrm{~mol} \mathrm{~L}^{-1}$ (Fenton's reaction) and $\mathrm{H}_{2} \mathrm{O}_{2} / \mathrm{Fe}^{2+} / \mathrm{UV} 0.10 / 0.08 \mathrm{~mol} \mathrm{~L}^{-1}$ (photo-Fenton reaction). The remediation was practically complete (99\%) after 30 min of the photo-Fenton process. No

(1) Recebido para publicação em 17 de maio de 2011 e aprovado em 25 de junho de 2012.

(2) Graduandas, Departamento de Química (DQ), Pontifícia Universidade Católica do Rio de Janeiro (PUC-RJ), Rua Marquês de São Vicente, 225, Gávea. CEP 22451-041 Rio de Janeiro (RJ), Brasil. E-mail: vagraciano@gmail.com, pires.carolina@gmail.com

(3) Pós-graduando, DQ, PUC-RJ. E-mail: scgteixeira@yahoo.com.br

(4) Pesquisadora, DQ, PUC-RJ. E-mail: rlziolli@puc-rio.br

(5) Pesquisador, Centro Nacional de Pesquisa de Solo, Embrapa Solos, Rua Jardim Botânico, 1024, Jardim Botânico. CEP 22460000 Rio de Janeiro (RJ), Brasil. E-mail: daniel@cnps.embrapa.br 
other oxidant reached this dissipation rate after $180 \mathrm{~min}$. The remediation was least efficient with $0.10 \mathrm{~mol} \mathrm{~L}^{-1} \mathrm{H}_{2} \mathrm{O}_{2}(70.51 \%)$. The addition of iron ions increased the dissipation rate to $86.98 \%\left(\mathrm{H}_{2} \mathrm{O}_{2} / \mathrm{Fe}^{2+} 0.10 / 0.08 \mathrm{~mol} \mathrm{~L} \mathrm{~L}^{-1}\right)$. The conventional methods reached rates of $76.58 \%$ $\left(\mathrm{KMnO}_{4} 0.10 \mathrm{~mol} \mathrm{~L}^{-1}\right)$ and $93.85 \%\left(\mathrm{~K}_{2} \mathrm{~S}_{2} \mathrm{O}_{8} 0.10 \mathrm{~mol} \mathrm{~L}^{-1}\right)$.

Index terms: Fenton reaction; peroxide; permanganate; persulfate.

\section{INTRODUÇÃO}

O solo é o destino de muitos compostos que podem alterar o equilíbrio e os ciclos naturais, compreendidos por isso como poluentes (Acioli et al., 2009; Teixeira et al., 2009). Um desses poluentes é o petróleo - fonte energética mais utilizada no mundo e matriz de muitos derivados importantes para vida moderna. A poluição por petróleo é de particular interesse devido à sua frequência de ocorrência tanto nos Estados Unidos (USEPA, 2007) quanto no Brasil (Cetesb, 2010); além disso, devem ser considerados seus efeitos carcinogênicos e genotóxicos (ATSDR, 1995), sendo nescessária a remediação rápida e completa desses sítios.

Existem diferentes métodos em que se usam reagentes químicos para remediar solos contaminados com petróleo, os quais são classificados como processos convencionais e, ou, oxidativos avançados. Os métodos convencionais são aqueles que usam íons como agentes oxidantes - por exemplo, o emprego de $\mathrm{KMnO}_{4}$. Os processos oxidativos avançados utilizam diferentes reagentes para produzir o radical hidroxila $(\cdot \mathrm{OH})$, uma espécie altamente oxidante e pouco seletiva. Exemplos desses métodos são os reagentes de Fenton $\left(\mathrm{H}_{2} \mathrm{O}_{2} / \mathrm{Fe}^{2+}\right)$ e foto-Fenton $\left(\mathrm{H}_{2} \mathrm{O}_{2} / \mathrm{Fe}^{2+} / \mathrm{luz}\right.$ ultravioleta artificial ou solar) (USEPA, 1998).

Os processos conhecidos como Fenton produzem o radical hidroxila $(\cdot \mathrm{OH})$ a partir da catálise do $\mathrm{H}_{2} \mathrm{O}_{2}$ com íons $\mathrm{Fe}$ em meio ácido, conforme a equação:

$$
\mathrm{H}_{2} \mathrm{O}_{2}+\mathrm{Fe}^{2+} \rightarrow \cdot \mathrm{OH}+\mathrm{OH}+\mathrm{Fe}^{3+}
$$

Além desses processos, em remediação de solos contaminados, ainda é possível aplicar o reagente persulfato de potássio $\left(\mathrm{K}_{2} \mathrm{~S}_{2} \mathrm{O}_{8}\right)$. Por meio da ação da temperatura $(\Delta)$, luz ou catálise de espécies metálicas $\left(\mathrm{M}^{\mathrm{n}+}\right)$, como o Fe ou a $\mathrm{Ag}$, é possível produzir o radical $\mathrm{SO}_{4}{ }^{\circ-}$ como espécie oxidante, segundo a reação (Liang et al., 2003):

$$
\begin{gathered}
\mathrm{S}_{2} \mathrm{O}_{8}{ }^{2-} \rightarrow 2 \mathrm{SO}_{4}{ }^{\cdot-}(\Delta \text { ou luz }) \\
\mathrm{S}_{2} \mathrm{O}_{8}{ }^{2-}+\mathrm{M}^{\mathrm{n}+} \rightarrow \mathrm{SO}_{4}{ }^{\cdot-}+\mathrm{SO}_{4}{ }^{2-}+\mathrm{M}^{(\mathrm{n}+1)+}
\end{gathered}
$$

São vários os trabalhos que aplicam processos oxidativos na descontaminação de solos (MacKinnon \& Thomson, 2002; Goi et al., 2006; Palmroth et al., 2006; Waldemer et al., 2007; Lee, 2008). Diversos são os motivos para que a remediação de solo por hidrocarbonetos de petróleo seja objeto de intensos estudos, e alguns deles podem ser citados. Os componentes dos solos interferem na eficiência dos processos, podendo aumentá-la ou diminuí-la (Acioli et al., 2009). Os próprios xenobióticos geralmente estão presentes como misturas, dificultando sua caracterização e a quantificação dos resultados. Somado a isso, os trabalhos que usam luz solar não têm repetibilidade, por que a intensidade da irradiação pode mudar até mesmo durante o curso do experimento. Por fim, o petróleo é constituído de substâncias tóxicas, recalcitrantes e, justamente em razão disso, em alguns casos, as técnicas de tratamento biológico e fitorremediação são inadequadas. Entretanto, se de um lado estudos comprovam a eficiência do uso de técnicas oxidativas para remoção desses poluentes do solo, na literatura científica há inúmeros trabalhos envolvendo diferentes categorias de solo, diversos métodos de oxidação e os mais diferentes objetivos; nessa diversidade de condições, torna-se difícil a comparação de eficiência entre esses trabalhos. Dessa maneira, este trabalho visou estudar o processo de remediação de um solo de maior distribuição geográfica do Brasill (Ker, 1997) contaminado com um tipo de petróleo, comparando a aplicação de diferentes agentes oxidantes.

\section{MATERIAL E MÉTODOS}

\section{Coleta do solo}

Uma amostra do horizonte A $(0-15 \mathrm{~cm})$ de Latossolo Vermelho-Amarelo foi coletada na cidade de Nova Friburgo, região serrana do Estado do Rio de Janeiro. O material foi seco ao ar e peneirado em malha de 0,40 mm. Ele foi coletado em setembro de 2006 e, depois de seco, destorroado e peneirado, foi guardado em caixas de papelão em ambiente seco, ao abrigo da luz.

\section{Caracterização físico-química e granulométrica}

Os métodos empregados encontram-se descritos em Embrapa (1997). A amostra demonstrou ser um solo ácido $(\mathrm{pH}=4,1)$, de textura franco-argiloarenosa (argila=260 $\mathrm{g} \mathrm{kg}^{-1}$; silte $=182 \mathrm{~g} \mathrm{~kg}^{-1}$; areia=558 $\mathrm{g} \mathrm{kg}^{-1}$ ), teor médio de carbono orgânico $\left(17,4 \mathrm{~g} \mathrm{~kg}^{-1}\right)$ e saturação por bases $=12 \%$.

\section{Contaminação do solo}

O solo seco e peneirado foi contaminado artificialmente com petróleo grau API=40 a uma concentração de $5.000 \mathrm{mg} \mathrm{kg}^{-1}$ solo, valor próximo ao encontrado em solos contaminados acidentalmente por derrame de petróleo, como no caso da REPAR - 
Refinaria Getúlio Vargas (Gogosz et al., 2010). Inicialmente, a massa de petróleo foi pesada diretamente em um balão volumétrico e dissolvida em diclorometano. A solução de petróleo foi vertida sobre o solo, que estava distribuído em placa de Petri. A solução de solo e petróleo foi homogeneizada manualmente, e o solvente, removido por evaporação ao abrigo da luz. O solo contaminado e seco foi devidamente acondicionado sob refrigeração, para posteriormente ser fracionado em partes iguais, para uso nos diversos experimentos.

\section{Teste de remediação via oxidação química}

Os ensaios de remediação do solo foram realizados pela adição da solução oxidante sobre o solo. Cada ensaio foi conduzido em erlenmeyer de vidro âmbar contendo $5 \mathrm{~g}$ do solo e $15 \mathrm{~mL}$ da solução oxidante, em três replicatas e nos tempos de 30, 60, 120 e 180 min. Os ensaios realizados foram: (i) oxidação com $\mathrm{KMnO}_{4}$ $0,10 \mathrm{~mol} \mathrm{~L}^{-1}$; (ii) oxidação com $\mathrm{K}_{2} \mathrm{~S}_{2} \mathrm{O}_{8} 0,10 \mathrm{~mol} \mathrm{~L}^{-1}$; (iii) $\mathrm{H}_{2} \mathrm{O}_{2} 0,10 \mathrm{~mol} \mathrm{~L}^{-1}$; (iv) $\mathrm{H}_{2} \mathrm{O}_{2} / \mathrm{Fe}^{2+} 0,10 / 0,080 \mathrm{~mol}$ $\mathrm{L}^{-1}$ (reação de Fenton); e (v) $\mathrm{H}_{2} \mathrm{O}_{2} / \mathrm{Fe}^{2+} / \mathrm{UV} 0,10 / 0,080$ mol L-1 (foto-Fenton). Nos ensaios de (i) até (iv) os erlenmeyers foram dispostos em um agitador mecânico de movimento circular, a uma velocidade de $250 \mathrm{rpm}$. Nos ensaios (v) foram utilizados béqueres de $100 \mathrm{~mL}$. O sistema foi fotoirradiado com o uso de uma lâmpada tubular de luz germicida de $40 \mathrm{~W}$ da Philips. As lâmpadas de luz germicida, com emissões máximas em 256 e $365 \mathrm{~nm}$, foram dispostas a uma altura de $25 \mathrm{~cm}$ sobre os béqueres. Estes, por sua vez, foram mantidos em agitação constante, de forma similar à dos demais experimentos. Os ensaios de oxidação foram conduzidos até que os extratos não apresentassem variação significativa no teor de compostos fluorescentes de petróleo extraíveis do solo - condição na qual se afirmou que cada método atingiu a sua capacidade máxima de oxidação nas condições experimentais estudadas.

\section{Monitoramento da degradação do petróleo}

Após agitação do sistema no tempo determinado, o sobrenadante foi separado da fase sólida por decantação, e a amostra de solo, submetida à extração em três etapas de $1 \mathrm{~h}$ cada, com alíquotas de $30 \mathrm{~mL}$ de diclorometano. A análise foi realizada por espectroscopia de fluorescência em espectrofluorímetro PerkinElmer, modelo LS 55 Luminescence Espectrometer. Os espectros foram obtidos em comprimento de onda de excitação de $250 \mathrm{~nm}$ e emissão variando de 278 a $483 \mathrm{~nm}$. Para a análise estatística, foi empregada a análise de variância, considerando um delineamento inteiramente casualizado, em que a fonte de variação foi o tipo de tratamento oxidativo, analisado para cada tempo. Em caso de ocorrência de diferença significativa, foi aplicado o teste de Tukey com nível de confiança de 0,05. Para isso, utilizou-se o software SAS versão 9.1.3.

\section{RESULTADOS E DISCUSSÃO}

Os resultados de degradação foram apresentados na figura 1 , que apresenta a relação de concentração de petróleo dividida pela concentração inicial $\left(\mathrm{C} / \mathrm{C}_{0}\right)$ em função do tempo de experimento.

No processo que contou com a aplicação de $\mathrm{H}_{2} \mathrm{O}_{2}$ como agente oxidante, foram removidos 70,51\% da carga inicial de petróleo em 180 min. Esse processo apresentou também a menor velocidade de reação (365 $\left.\mathrm{mg} \mathrm{kg}^{-1} \mathrm{~min}^{-1}\right)$.

$\mathrm{O}$ processo convencional usando $\mathrm{KMnO}_{4}$ como agente oxidante apresentou taxa de degradação de $76,58 \%$ em 180 min. Esse processo também mostrou uma das menores velocidades de reação, na faixa de $369 \mathrm{mg} \mathrm{kg}^{-1} \mathrm{~min}^{-1}$.

A análise estatística por meio do teste $\mathrm{F}$ dos resultados obtidos com $\mathrm{H}_{2} \mathrm{O}_{2}$ e $\mathrm{KMnO}_{4}$ não indicou diferença significativa entre os resultados obtidos pelas duas técnicas ( $\mathrm{F}$ tabelado $=7,71$; $\mathrm{F}$ calculado $=0,84)$.

$\mathrm{O}$ processo em que se utilizou $\mathrm{K}_{2} \mathrm{~S}_{2} \mathrm{O}_{8}$ apresentou velocidade de reação de $484 \mathrm{mg} \mathrm{kg}^{-1} \mathrm{~min}^{-1}$ e taxa de degradação de 93,85 \% em 180 min.

Os processos conhecidos como Fenton foram os mais rápidos e eficientes, comparados com os outros tipos de reação. Em apenas $30 \mathrm{~min}$, os processos de Fenton $\left(\mathrm{H}_{2} \mathrm{O}_{2} / \mathrm{Fe}^{2+} 0,10 / 0,080 \mathrm{~mol} \mathrm{~L}^{-1}\right)$ e foto-Fenton $\left(\mathrm{H}_{2} \mathrm{O}_{2} / \mathrm{Fe}^{2+} / \mathrm{UV} 0,10 / 0,080 \mathrm{~mol} \mathrm{~L}^{-1}\right)$ atingiram a sua capacidade máxima de remoção da carga poluidora dentro das condições experimentais. O processo de Fenton foi capaz de remover 86,98\% em $180 \mathrm{~min}$.

O processo de foto-Fenton foi o mais eficiente: em 120 min removeu o total de $99,33 \%$ da carga total de petróleo adicionada ao solo.

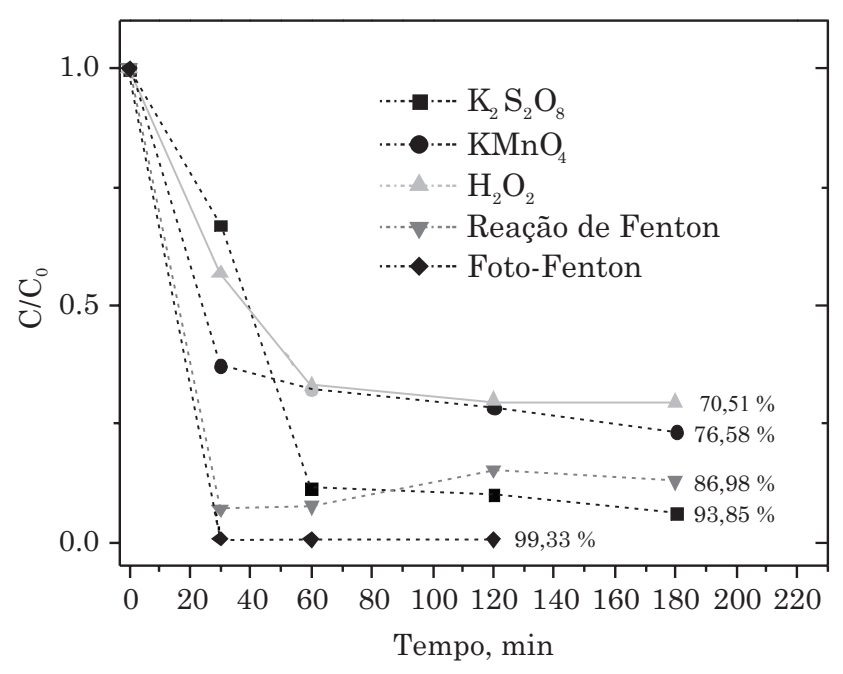

Figura 1. Taxas de remediação de petróleo em Latossolo Vermelho-Amarelo. 
No período total de 180 min, o processo de Fenton apresentou velocidade de remediação de $1.015 \mathrm{mg} \mathrm{kg}^{-1} \mathrm{~min}^{-1}$, enquanto o processo de fotoFenton apresentou velocidade superior de $1.085 \mathrm{mg}$ $\mathrm{kg}^{-1} \mathrm{~min}^{-1}$. Esta velocidade foi superior à de todos os demais processos aplicados, quando avaliados no mesmo período de tempo.

Comparando-se o processo usando o reagente de Fenton com aquele em que se aplicou $\mathrm{K}_{2} \mathrm{~S}_{2} \mathrm{O}_{8}$ por meio da análise estatística por teste $\mathrm{F}$, novamente não foi observada diferença significativa entre as técnicas $(\mathrm{F}$ tabelado $=7,71 ; \mathrm{F}$ calculado $=6,67)$.

Os estudos de foto-Fenton não foram conduzidos até 180 min justamente por não apresentarem sinal fluorescente detectável a partir de $30 \mathrm{~min}$. Na figura 1, observa-se aumento da concentração extraível de petróleo do solo pelo aumento do valor de $\mathrm{C} / \mathrm{C}_{0}$. $\mathrm{A}$ reação de Fenton é exotérmica, e o calor gerado facilitou a dessorção do petróleo do solo. O mesmo não foi observado no experimento de foto-Fenton, uma vez que a fração fluorescente do petróleo foi degradada.

Ao comparar os processos usando $\mathrm{H}_{2} \mathrm{O}_{2}$, Fenton $\left(\mathrm{H}_{2} \mathrm{O}_{2} / \mathrm{Fe}^{2+}\right)$ e foto-Fenton $\left(\mathrm{H}_{2} \mathrm{O}_{2} / \mathrm{Fe}^{2+} / \mathrm{UV}\right)$, observaram-se aumento progressivo das taxas de remoção da carga poluidora e na velocidade de reação em função da adição de íons $\mathrm{Fe}^{2+}$ e luz ultravioleta ao processo. A presença de $\mathrm{Fe}$ levou à formação do radical hidroxila, e a presença da luz acelerou a formação do radical e o processo de remediação, consequentemente. Esse resultado sugere que a geração de radicais hidroxila propiciou melhores condições de oxidação do petróleo.

No quadro 1 é apresentado um comparativo do potencial de oxidação das espécies oxidantes utilizadas neste trabalho.

Os reagentes $\mathrm{H}_{2} \mathrm{O}_{2}$ e $\mathrm{KMnO}_{4}$ apresentam potenciais de oxidação semelhantes $(1,8$ e $1,7 \mathrm{~V}$, respectivamente) e os menores em comparação aos demais agentes utilizados. Comparando o potencial desses dois oxidantes com os resultados obtidos, esses agentes mostraram as menores taxas de remediação do solo nas condições estudadas, em razão dos menores potenciais de oxidação.

Esses reagentes apresentam também algumas desvantagens do ponto de vista de estabilidade química

Quadro 1. Potencial de oxidação das espécies oxidantes $^{(1)}$ utilizadas na remediação do solo estudado

\begin{tabular}{lc}
\hline Espécie oxidante & Potencial de oxidação $(\mathrm{V})$ \\
\hline Radical hidroxila & 2,8 \\
Radical sulfato & 2,6 \\
$\mathrm{H}_{2} \mathrm{O}_{2}$ & 1,8 \\
$\mathrm{KMnO}_{4}$ & 1,7 \\
\hline
\end{tabular}

(1) (Liang et al., 2004). e toxidez ambiental. $\mathrm{O}_{2} \mathrm{O}_{2}$ é um reagente instável quando não mantido em baixas temperaturas ou fora das suas condições de armazenamento. É conveniente lembrar que a utilização de $\mathrm{KMnO}_{4}$ pode deixar resíduos de Mn no solo.

\section{CONCLUSÕES}

1. O reagente de foto-Fenton foi o mais eficiente, removendo $99,33 \%$ da carga poluidora.

2. $\mathrm{O}$ uso de $\mathrm{K}_{2} \mathrm{~S}_{2} \mathrm{O}_{8}$ é uma alternativa promissora para remediação de solos contaminados com petróleo, sendo capaz de remediar 93,85 \% da carga poluidora adicionada ao solo.

3. A aplicação de radiação ultravioleta teve papel primordial no aumento da eficiência dos processos que utilizaram $\mathrm{H}_{2} \mathrm{O}_{2}$.

\section{AGRADECIMENTO}

Gentilmente agradecemos ao Prof. Dr. Ricardo Aucélio de Queiroz, Departamento de Química da PUC-Rio, pelo uso do espectrofluorímetro utilizado nesse trabalho.

\section{LITERATURA CITADA}

ACIOLI, C.; AGUIAR, M.R.M.P. \& PÉREZ, D.V. Avaliação da potencialidade de processos pseudo-fenton para remediação de solos contaminados por diesel. Quím. Nova, 32:2000-2002, 2009.

AGENCY FOR TOXIC SUBSTANCES AND DISEASE REGISTRY - ATSDR. Toxicological Profile for Polycyclic Aromatic Hydrocarbons (PAHs). Atlanta, U.S. Department of Health \& Human Services, 1995. Disponível em: <http://www.atsdr.cdc.gov/toxprofiles/tp69.pdf>. Acesso em : 19 de out. de 2010.

COMPANHIA DE TECNOLOGIA DE SANEAMENTO AMBIENTAL - CETESB. Áreas Contaminadas. Disponível em: <http://www.cetesb.sp.gov.br/Solo/ areas_contaminadas/texto_areas_cont_nov_08.pdf $>$. Acesso em: 19 de out. de 2010.

EMPRESA BRASILEIRA DE PESQUISA AGROPECUÁRIA EMBRAPA. Centro Nacional de Pesquisa do Solo. Manual de métodos de análises de solo. 2.ed. Rio de Janeiro, 1997. 212p.

GOGOSZ, A.M.; BONA, C.; SANTOS, G.O. \& BOTOSSO, P.C. Germination and initial growth of Campomanesia xanthocarpa O. Berg. (Myrtaceae), in petroleumcontaminated soil and bioremediated soil. Braz. J. Biol., 70:977-986, 2010 . 
GOI, A.; KULIK, N. \& TRAPIDO, M. Combined chemical and biological treatment of oil contaminated soil. Chemosphere, 63:1754-1763, 2006.

KER, J.C. Latossolos do Brasil: Uma revisão. Geonomos, 5:1740, 1997.

LEE, R.F. Photo-oxidation and photo-toxicity of rude and refined oils. Spill. Sci. Technol. B., 8:157-162, 2008.

LIANG, C.; BRUELL, C.J.; MARLEY, M.C. \& SPERRY, K.L. Persulfate oxidation for in situ remediation of TCE.II. Activated by chelated ferrous ion. Chemosphere, 55:12251233, 2004.

LIANG, C.; BRUELL, C.J.; MARLEY, M.C. \& SPERRY, K.L. Thermally activated persulfate oxidation of trichloroethylene (TCE) and 1,1,1-trichloroethane (TCA) in aqueous and soil slurry systems. Soil Sediment Contam., 12:207-228, 2003.

MacKINNON, L.K. \& THOMSON, N.R. Laboratory-scale in situ chemical oxidation of a perchloroethylene pool using permanganate. J. Contam. Hydrol., 56:49-74, 2002 .
PALMROTH, M.R.T.; LANGWALDT, J.H.; AUNOLA, T.A.; GOI, A.; MÜNSTER, U.; PUHAKKA, J.A. \& TUHKANEN, T.A. Effect of modified Fenton's reaction on microbial activity and removal of PAHs in creosote oil contaminated soil. Biodegradation, 17:29-39, 2006.

TEIXEIRA, S.C.G.; DE AGUIAR, M.R.M.P.; CANELA, M.C.; ZIOLLI, R.L. \& PÉREZ, D.V. Study of pyrene adsorption on brazilian soils. R. Chim., 60:583-587, 2009.

UNITED STATES ENVIRONMENTAL PROTECTION AGENCY - USEPA. Advanced Photochemical Oxidation Processes (EPA/625/R-98/004). Washington DC, United States Environmental Protection Agency, 1998.

UNITED STATES ENVIRONMENTAL PROTECTION AGENCY - USEPA. Treatment technologies for site cleanup: Annual Status Report (ASR). Twelfth Edition (EPA 542-R-07-012). Washington DC, United States Environmental Protection Agency, 2007.

WALDEMER, R.H.; TRATNYEK, P.G.; JOHNSON, R.L. \& NURMI, J.T. Oxidation of chlorinated ethenes by heatactivated persulfate: Kinetics and products. Environ. Sci. Technol., 41:1010-1015, 2007. 